\title{
An alternative parameterisation for binary-lens caustic-crossing events
}

\author{
A. Cassan \\ Astronomisches Rechen-Institut (ARI), Zentrum für Astronomie (ZAH), Heidelberg University, Mönchhofstraße 12-14, \\ 69120 Heidelberg, Germany \\ e-mail: cassan@ari.uni-heidelberg.de
}

Received 18 March 2008 / Accepted 16 August 2008

ABSTRACT

\begin{abstract}
Aims. Microlensing events are being discovered and alerted by the two survey teams OGLE and MOA at an increasing rate. Around ten percent of these events involve binary lenses. Such events potentially contain much information on the physical properties of the observed binary systems, which can then be used for e.g. statistical studies on binary objects in the Galactic disk or bulge. However, such events are usually not straightforward to study, because the model equations are strongly non-linear and there are many local minima that can fool the search for the best solution if the parameter space is not inspected with great care. In this work an alternative parameterisation for the binary lens fitting problem is proposed, in which the parameters involved are defined to represent as closely as possible the caustic-crossing features observed in most binary lens light curves. Furthermore, we work out an extension of the method in order to make use of the straight line fold caustic approximation, when the latter applies for both the caustic entry and exit. Methods. We introduce an alternative parameterisation in order to confine the exploration of the parameter space to regions where the models only involve caustic crossing at the dates seen in the light curve.

Results. We find that the proposed parameterisation provides more robustness to the light curve fitting process, in particular in avoiding a code to get stuck in false minima.
\end{abstract}

Key words. gravitational lensing - methods: numerical - methods: analytical

\section{Introduction}

Since the original idea of Paczynski (1986) to use the gravitational lensing effect to track the presence of dark objects in the Milky Way, more than three thousand microlensing events have been discovered, mostly in the direction of the Galactic centre. A microlensing event occurs when the light from a background bright star (or source) is bent by the gravitational field of an intervening massive body (or lens) passing close to the observer lineof-sight. The phenomenon produces an apparent transient brightening of the source flux, resulting in a typical bell-shaped light curve. Current surveys operated by the OGLE (Udalski 2003) and MOA (Bond et al. 2001) collaborations provide lists of ongoing microlensing events by monitoring more than $10^{8}$ stars towards the Galactic bulge on a daily basis, while (up to the 2007 bulge season) collaborations like PLANET, RoboNet or $\mu$ FUN use ground-based networks of telescopes to perform a round-the-clock follow-up of selected events.

Since the information on the lens characteristics is contained in the lens mass distribution rather than in its luminosity, microlensing naturally opens a new window in the exploration of invisible objects within the Galaxy. Indeed, shortly after Paczynski (1986)'s work, Mao \& Paczynski (1991) proposed microlensing as a promising tool to hunt for extrasolar planets, a few years before the first planets were discovered around a pulsar (Wolszczan \& Frail 1992) by pulse timing, and around a solar-like star (Mayor \& Queloz 1995) by radial velocity measurements.

Searching for planets is today the leading motivation of Galactic microlensing observations. Amongst the $~ 300$ exoplanets discovered so far, most of them using the radial velocity technique, microlensing contributes seven planets. Four of them are giant planets of a few Jupiter masses: MOA 2003-BLG53/OGLE 2005-BLG-235Lb (Bond et al. 2004), OGLE 2005BLG-071Lb (Udalski et al. 2005; Dong et al. 2008), plus a slightly less massive Saturn/Jupiter planetary system analogue (OGLE 2007-BLG-109Lb+c, Gaudi et al. 2008), orbiting lowmass dwarf stars. A similar number of super-Earth planets have been found as well: OGLE 2005-BLG-390Lb (Beaulieu et al. 2006; Kubas et al. 2008), a 5.5 Earth-mass planet at 2.6 AUs which makes it the first ever rocky/icy planet discovered around a main sequence host beyond the snow line, OGLE 2005-BLG169Lb (Gould et al. 2006), 13 times more massive than Earth, and recently MOA 2007-BLG-192Lb (Bennett et al. 2008), one of the least massive planet discovered so far with an estimated mass of only $\sim 3 M_{\oplus}$, probably orbiting a very late $\mathrm{M}$ or brown dwarf.

While microlensing has so far contributed little to the large number of extrasolar planet discoveries, it probes a region in the mass/orbit diagram - namely cool low-mass planets (cold Neptunes and super-Earths) at few AUs from their stars - where no other competitive technique has the required sensitivity. Furthermore, microlensing is sensitive to extremely low mass bodies, so that planets of mass below that of the Earth could be reached by a dedicated space mission (Bennett \& Rhie 2002).

Most of the microlensing events detected do not show evidence of the presence of planets around the lenses. For these so-called single lens events, modelling is relatively easy and it is possible to use a large number of events to draw statistical conclusions on the abundance of planets. Such studies have been carried out in particular by Gaudi et al. (2002) for a selection of PLANET events in the period 1995-1999, Tsapras et al. (2003) with a sample based on OGLE events between 1998-2000, and Snodgrass et al. (2004) using data form the OGLE 2002 
campaign. Nevertheless, the nature of many anomalous events remains unclear because their modelling requires much more work and computational resources. Up to now, such events have led to separate studies in order to derive constraints on the presence of planets around the lens, as e.g. performed for the very high magnification event (i.e. very sensitive to planetary signals) OGLE 2007-BLG-050 (Batista et al. 2008).

The steadily increasing number of anomalous microlensing events is thus posing a great challenge to modellers, for the proper treatment of an individual case requires one to probe many models, hence demanding very efficient modelling schemes based on robust mathematical and numerical methods. A large fraction of binary lens events become unmodelled each season. Since these represent close to $10 \%$ (Jaroszynski et al. 2004) of the overall sample (close to a thousand events a year), a special effort to provide improved and more automated modelling strategies is needed.

Events that exhibit caustic crossings are usually very rewarding in terms of extracting stronger constraints on the physics of the binary lens system, such as the masses and physical separations of the individual components (e.g. Jaroszynski et al. 2005; Kubas et al. 2005). The first microlensing event showing observational evidence of a binary lens caustic crossing is OGLE No. 7 (Udalski et al. 1994), and the first planet discovered by microlensing MOA 2003-BLG-53/OGLE 2005-BLG-235Lb (Bond et al. 2004) also involves a caustic crossing, emphasing the importance of such binary lens events.

Although the light curve features associated with caustic crossings can be very pronounced and easily recognised, the underlying best model can be hard to find since the parameter space often is afflicted by many local minima, even if the light curve is well covered. This problem is aggravated by using parameterisations in which the trajectory is modelled by parameters that are not well related to the light curve features. This is for example the case in the widely used binary lens standard parameterisation using the angle between the binary lens axis and the minimum distance between the trajectory and the origin of the coordinate system. A main reason for this is that the equations of lensing are strongly non-linear and changing a given trajectory parameter can have very unexpected consequences for the shape of the light curve. This is explained by the presence of extended gravitational caustics when the lens is composed of more than one body. When the source crosses a caustic, its flux experiences a very steep magnification gradient. Hence, even a small change in the trajectory can cause a very strong change in flux. A desired characteristic of an alternative parameterisation would be to directly relate the model parameters to visible features in the light curve, such as the caustic crossing dates, whatever the source trajectory. All probed configurations in the minimisation process would then display caustic crossings at their observed position on the light curve. We aim here to provide an alternative parameterisation of the binary lens caustic crossing model that works in this way.

The paper is organised as follows: in Sect. 2, we introduce the basic principles of the method and describe the new parameterisation, in particular the choice of a curvilinear abscissa along the caustic lines. In Sect. 3 we detail the implementation of the method, and discuss the possible fitting strategies and the inclusion of more elaborate binary lens models within this framework. We also tackle some important aspects arising from the introduced set of parameters. In Sect. 5, we work out in detail a possible way to implement the so-called straight line fold caustic approximation within the presented framework, which allows under some assumptions extremely efficient computations. We summarise in Sect. 6 our main results, and discuss the broader implication of fitting schemes and strategies to provide more automated modelling software in the context of future generations of robotic telescopes.

\section{The method}

\subsection{Caustic curves}

The fundamental equation of Galactic microlensing is the lens equation, which links the angular position of a point-source object to the angular positions of its multiple images in units of $\theta_{\mathrm{E}}$, the angular Einstein radius (Einstein 1936):

$\theta_{\mathrm{E}}=\sqrt{\frac{4 G M}{c^{2}}\left(\frac{D_{\mathrm{S}}-D_{\mathrm{L}}}{D_{\mathrm{S}} D_{\mathrm{L}}}\right)}$,

with $D_{\mathrm{S}}, D_{\mathrm{L}}$ the observer-source and -lens distances respectively, and $M$ the lens total mass. In the formalism of Witt (1990) used here, the binary lens equation is written using the complex notation:

$\zeta=z-\frac{1}{1+q}\left(\frac{1}{\bar{z}}+\frac{q}{\bar{z}+d}\right)$

where we have chosen the coordinate system so that the more massive component of the lens is located at the origin, with the secondary body located on the left at a separation ${ }^{1} d$. Finally, $q \leq 1$ denotes the binary lens mass ratio.

Multiple lenses in general harbour caustic lines, which are imaginary lines on the plane of the sky where the magnification of a perfect point-source diverges. Mathematically, they draw closed curves where the Jacobian of the transformation $z \rightarrow \zeta$ vanishes. In the following and for brevity, we will refer to the term "caustic" not only for the lines themselves, but also for the closed curves they form. In the case of binary lenses, caustics exist in three different topologies, usually referred as close, intermediate and wide binaries (see Dominik 1999b, for an explicit discussion about these limits for extreme mass ratios), which are illustrated in Fig. 1. In the close binary case (on the left in the figure), there exits a central caustic near the more massive body, as well as two secondary caustics ${ }^{2}$ above and below the binary symmetry axis. The intermediate binary configuration (in the middle) displays a single caustic (also called a resonant caustic when $d \sim 1$ ), while the wide binary (on the right) involves a central caustic plus an isolated single secondary caustic, located between the two lens components.

It has been shown by Erdl \& Schneider (1993) in the mathematical framework of catastrophe theory that there exist close analytical formulae which determine the bifurcation values between these regimes, which only depend on the lens separation and mass ratio $(d, q)$ :

$d_{\mathrm{c}}^{8}=\frac{(1+q)^{2}}{27 q}\left(1-d_{\mathrm{c}}^{4}\right)^{3}$,

$d_{\mathrm{w}}^{2}=\frac{\left(1+q^{1 / 3}\right)^{3}}{1+q}$,

where $d_{\mathrm{c}}(q)$ and $d_{\mathrm{w}}(q)$ are respectively delimiting the closeintermediate and intermediate-wide binary regimes for a given value of the mass ratio $q$. The resulting frontiers are plotted in Fig. 1.

\footnotetext{
1 The term "separation" has to be understood as the projected distance between the two lens components on the plane of the sky, in units of $\theta_{\mathrm{E}}$. ${ }^{2}$ In the case of planetary mass ratios $(q \ll 1)$, secondary caustics are usually referred as "planetary" caustics.
} 


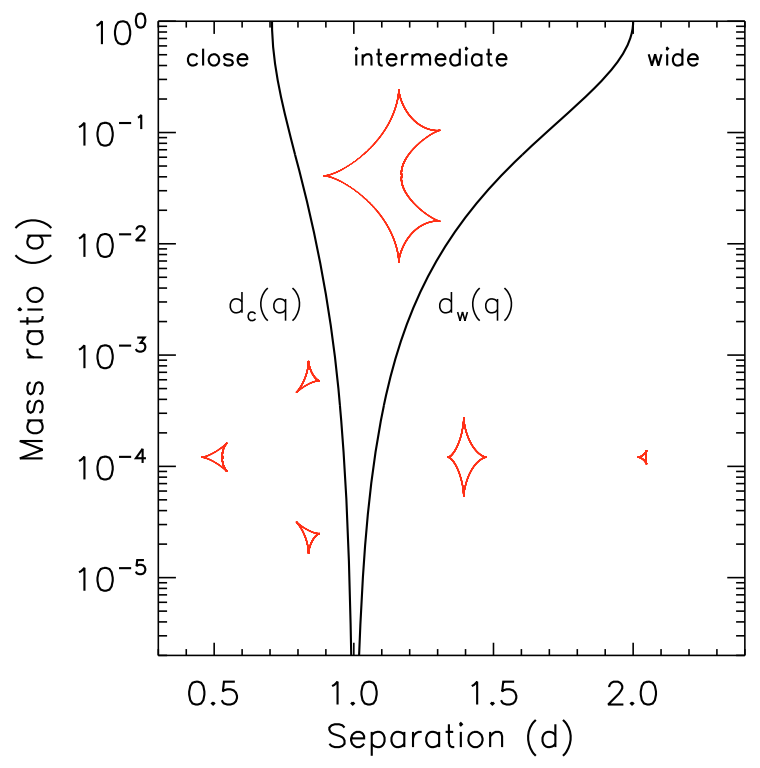

Fig. 1. The three topologies of binary lenses: the close binary lens (on the left), which involves a central caustic plus two off-axis small secondary caustics (plotted here for $d=0.8$ and $q=10^{-2}$ ), the intermediate binary (in the middle, assuming $d=1$ and $q=10^{-2}$ ) with a single caustic, and the wide binary (on the right, $d=1.6$ and $q=10^{-2}$ ) whith a central caustic and an isolated secondary caustic. The figure also shows the bifurcation functions $d_{\mathrm{c}}(q)$ and $d_{\mathrm{w}}(q)$ between the three regimes, which are respectively displayed from left to right as the two vertical black lines. When the mass ratio tends to zero, the extension of the intermediate domain also tends to zero.

\subsection{The binary lens parameterisation}

In the simplest case, modelling a binary-lens microlensing event requires seven parameters, which are classically the lens separation $d$ and mass ratio $q$, the time $t_{0}$ of the closest approach of the source to the origin (here the primary body), $u_{0}$ the corresponding impact parameter in units of $\theta_{\mathrm{E}}, t_{\mathrm{E}}$ the Einstein crossing time, $\alpha$ the angle of the trajectory with respect to the $x$-axis, and the source radius $\rho_{*}$ in units of $\theta_{\mathrm{E}}$. The corresponding parameterisation is illustrated in Fig. 2.

While this straightforward parameterisation (hereafter referred to as "classical") is very well suited to describe single lens or non-caustic crossing binary lens events, it suffers from being rather disconnected from observable features in caustic-crossing light curves. The main reason is that the corresponding parameters contain no information on where the source crosses the caustics. As a consequence, widely exploring the classical parameter space in caustic-crossing events poses an important computational challenge. Firstly, because a large proportion of the tested models do not produce caustic crossings located at the observed position on the light curve, and only a fraction of the calculated models contribute efficiently in the minimising numerical procedure to find the solution. Secondly, caustic-crossing events are known to exhibit very uneven $\chi^{2}$ hyper-surfaces (see such a $\chi^{2}$ map in e.g. Kubas et al. 2005), and usually harbour a large number of local minima which should be found and considered as competitive models to explain the light curve (e.g. Dominik 1999a). Furthermore, in standard minimising routines, parameters vary within bounded intervals or around starting values, which may cause the routines to become stuck in a false minima if one (or more) of the parameters is not correctly optimised. Hence, parameterisations oriented towards observed features in the light

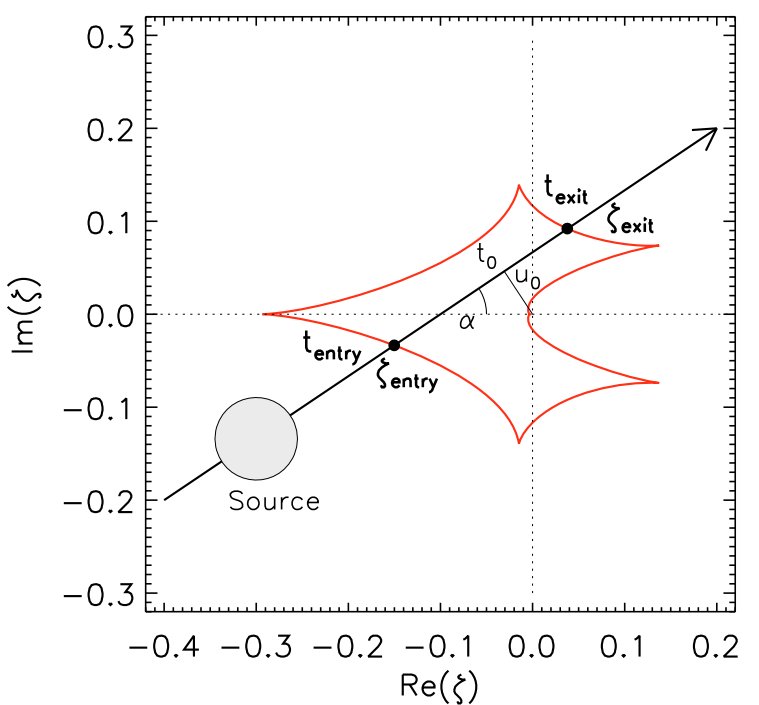

Fig. 2. A binary lens generates caustics, as displayed here (red lines) for a system of two masses located on the $x$-axis with separation $d=1$ and mass ratio $q=10^{-2}$ (intermediate binary lens). The more massive (or primary) body is located at the origin of the coordinate system, while the lighter component is on the left. In the simplest case, the source centre trajectory is classically described (in thin letters) by a straight line that passes at time $t_{0}$ at the minimum distance $u_{0}$ from the coordinate system, and makes an angle $\alpha$ with respect to the $x$-axis. In the proposed alternative parameterisation (in bold face), the source motion is expressed by means of the caustic entry and exit dates $t_{\text {entry }}$ and $t_{\text {exit }}$, which occur at positions $\zeta_{\text {entry }}\left(s_{\text {entry }}\right)$ and $\zeta_{\text {exit }}\left(s_{\text {exit }}\right)$ on the caustics. The corresponding model parameters $s_{\text {entry }}$ and $s_{\text {exit }}$ result from a proper definition of a curvilinear abscissa along the caustic lines (cf. Sect. 2.3).

curve help to circumvent strong correlations and parameter degeneracies. Additionally, a set of different starting points should be considered to ensure the completeness of the parameter space exploration.

One of the requirements when building the new parameterisation is that the number of parameters should remain the same as for the classical one, but with parameters which reflect as much as possible the caustic crossing features that are seen in the light curve. Albrow et al. (1999) proposed a parameterisation adapted to model a light curve where one caustic crossing is clearly identified in the light curve, and for which a straight line caustic approximation can be applied. In the general context discussed in this section, we keep to the more general case where finite source effects are calculated with no approximation, because the new parameterisation only affects the geometry of the problem and not the way to compute the magnification (see Sect. 4.1 for more details). Nevertheless, we examine in Sect. 5 the inclusion of the straight line caustic approximation in our framework, thus extending the work initiated by Albrow et al. (1999).

We therefore introduce in place of the four parameters $u_{0}, \alpha$, $t_{\mathrm{E}}$ and $t_{0}$, the dates of the caustic crossing at the entry and exit dates, $t_{\text {entry }}$ and $t_{\text {exit }}$, as well as the two corresponding curvilinear abscissa $s_{\text {entry }}$ and $s_{\text {exit }}$ along the caustic, so that $\zeta_{\text {entry }}=\zeta\left(s_{\text {entry }}\right)$ and $\zeta_{\text {exit }}=\zeta\left(s_{\text {exit }}\right)$ are the points in the source plane where the trajectory intersects the caustic (cf. Fig. 2). Within a suitable range for these four parameters, a theoretical light curve will display caustic crossing features around the observed entry and exit dates. The geometric conversion between the two set of parameters is straightforward, since $\zeta_{\text {entry }}$ and $\zeta_{\text {exit }}$ define the direction of the source motion. 


\subsection{Curvilinear abscissa along the caustic curve}

In order to define a proper curvilinear abscissa, ones needs to build an appropriate parameterisation of the caustic curves. A possible option is to follow the method initiated by Erdl \& Schneider (1993), for whom the caustics are described in a polar coordinate system $(r, \phi)$ in the source plane. One then needs to solve for $\cos (\phi)$ for a given value of $r$, hence parameterising the caustic line by $r$. A working implementation of such a parameterisation is described in detail by Dominik (2007) in the context of image contouring.

In this paper, we work out an alternative method, based on a result obtained by Witt (1990): for a point-source $\zeta$ exactly located on a caustic, its corresponding point-like image $z$ on the critical curve in the lens plane satisfies the equation:

$\frac{1}{1+q}\left[\frac{1}{z^{2}}+\frac{q}{(z+d)^{2}}\right]=\mathrm{e}^{-\mathrm{i} \phi}$,

where $\phi \in[0,2 \pi]$ is an ad hoc parameter, independent of the choice of the coordinate system. For a given binary lens $(d, q)$ configuration, we can compute for every $\phi$ in the range $0-2 \pi$ the caustic points by writing Eq. (5) as a complex polynomial of fourth degree:

$z^{4}+2 d z^{3}+\left(d^{2}-\mathrm{e}^{\mathrm{i} \phi}\right) z^{2}-\frac{2 d \mathrm{e}^{\mathrm{i} \phi}}{1+q} z-\frac{d^{2} \mathrm{e}^{\mathrm{i} \phi}}{1+q}=0$.

For a given $\phi$, this equation has four distinct complex solutions, which means the caustics are entirely built when $\phi$ runs from 0 to $2 \pi$. Moreover, since the caustics never intersect in the case of a binary lens (contrary to a triple lens for example), there exists a unique value of $\phi$ for a given $\zeta$ on the caustic (otherwise one would have $\zeta\left(\phi_{1}\right)=\zeta\left(\phi_{2}\right)$ with $\phi_{1} \neq \phi_{2}$, which would mean an intersection of the caustic lines). In this respect, Eq. (5) is optimal in the sense that each solution of the equation maps one and only one point on the caustic lines, and the caustics are fully drawn for $0 \leq \phi<2 \pi$.

We now examine how the caustics are drawn with $\phi$ for each of the three topologies. Since the caustics are symmetric with respect to the $x$-axis, we restrict ourselves to $\mathfrak{J}(\zeta) \geq 0$. By doing so, only two of the four solutions of Eq. (5) are taken into account. The caustic region where $\mathfrak{J}(\zeta)<0$ is built by symmetry afterwards. The corresponding caustics are drawn in Fig. 3, to which the reader is referred in the following.

- In the intermediate binary case, $\phi=0$ leads to three points, $\mathrm{A}-\mathrm{C}$. When $\phi$ increases to $2 \pi$, the caustics are simultaneously drawn by two distinct branches: $\mathrm{A} \rightarrow \mathrm{C}$ and $\mathrm{C} \rightarrow \mathrm{B}$.

- In the wide binary case, $\phi=0$ correspond to the four points $\mathrm{A}-\mathrm{D}$. When $\phi$ increases to $2 \pi$, the central caustic is built with the single branch $\mathrm{C} \rightarrow \mathrm{B}$, while at the same time the secondary caustic is built by the branch $\mathrm{A} \rightarrow \mathrm{D}$.

- In the close binary case, $\phi=0$ correspond to A, D and C. When $\phi$ increases to $2 \pi$, the central caustic is built with the single branch $\mathrm{A} \rightarrow \mathrm{D}$, while at the same time the single branch $\mathrm{C} \rightarrow \mathrm{C}$ draws the full secondary upper caustic (so that no horizontal reflection is needed). The lower secondary caustic is then the symmetric image of the upper one.

A first naive choice would be to simply take $\phi$ as a curvilinear abscissa. But, as shown in the upper panel on the left of Fig. 3, a regular sampling on $\phi$ (i.e. $\delta \phi=$ const.) results in a very irregular linear sampling of the caustics. Most of the points accumulate near the cusps, which makes this parameter not well-suited. To avoid this effect, we define a curvilinear abscissa $s$ which satisfies the property: $\delta s=$ const. $\Rightarrow|\zeta(s+\delta s)-\zeta(s)|=$ const.'. Since at first order $\zeta(s+\delta s) \simeq \zeta(s)+(\mathrm{d} \zeta / \mathrm{d} s) \delta s$, the condition to be fulfilled reads: $|\mathrm{d} \zeta / \mathrm{d} s|=K$, with $K$ a constant, so that:

$\left|\frac{\mathrm{d} \zeta}{\mathrm{d} \phi}\right|=\left|\frac{\mathrm{d} \zeta}{\mathrm{d} s}\right| \times\left|\frac{\mathrm{d} s}{\mathrm{~d} \phi}\right|=K \times\left|\frac{\mathrm{d} s}{\mathrm{~d} \phi}\right|$.

The abscissa $s$ can finally be obtained as a function of $\phi$ by integrating the derivative $\mathrm{d} s / \mathrm{d} \phi$, choosing $s(\phi)$ monotonic:

$s(\phi)=\int_{0}^{\phi} \frac{\mathrm{d} s}{\mathrm{~d} \phi} \mathrm{d} \phi+s(0)=\frac{1}{K} \int_{0}^{\phi}\left|\frac{\mathrm{d} \zeta}{\mathrm{d} \phi}\right| \mathrm{d} \phi+s(0)$,

where the normalisation constant $K$ depends on the choice of the ranges for $\phi$ and $s$. This is discussed in detail in Sect. 3 for the three topologies. A remaining technical point here concerns the calculation of the derivative $\mathrm{d} \zeta / \mathrm{d} \phi$. By differentiating Eq. (2) with respect to $\phi$ and substituting Eq. (5), we first obtain:

$\frac{\mathrm{d} \zeta}{\mathrm{d} \phi}=\frac{\mathrm{d} z}{\mathrm{~d} \phi}+\mathrm{e}^{\mathrm{i} \phi} \frac{\mathrm{d} \bar{z}}{\mathrm{~d} \phi}$

we then get the expression of $\mathrm{d} z / \mathrm{d} \phi$ by writing

$\frac{\mathrm{d}}{\mathrm{d} \phi} \mathrm{e}^{-\mathrm{i} \phi}=\frac{\mathrm{d} z}{\mathrm{~d} \phi} \times \frac{\mathrm{d}}{\mathrm{d} z} \mathrm{e}^{-\mathrm{i} \phi}=-\mathrm{i} \mathrm{e}^{-\mathrm{i} \phi}$,

which leads, after differentiating Eq. (5) with respect to $z$, to

$\frac{\mathrm{d} z}{\mathrm{~d} \phi}=\frac{\mathrm{i}}{2} \frac{(z+d)^{2}+q z^{2}}{(z+d)^{3}+q z^{3}}(z+d) z$,

and so $\mathrm{d} \zeta / \mathrm{d} \phi$ can be calculated analytically given a point $\zeta$ on a caustic (i.e. a point $z$ on a critical curve).

In Fig. 3, with the exception of the upper panel on the left commented on previously, we have used a regular sampling of $s$ to draw the caustic lines, following the above prescriptions. One can notice the regular spacing of the points along the caustics, even for the small number of sampling points chosen here (130 for a whole caustic). When $\phi$ runs between 0 and $2 \pi$, it draws a caustic which may contain cusp singularities. Because of the nature of such a singularity, the tangential vector to the curve vanishes (e.g. Witt 1990), and thus the derivative of $\zeta$ satisfies at the cusps locations: $(\mathrm{d} \zeta / \mathrm{d} \phi)_{\text {cusp }}=0$, or according to Eq. (7), $(\mathrm{d} s / \mathrm{d} \phi)_{\text {cusp }}=0$. The cusps can be seen in Fig. 3 at the location of the inflexion points of $s(\phi)$.

\section{Implementation}

As seen in the previous section, the caustics are built by gathering different branches obtained by moving $\phi$ from 0 to $2 \pi$. In our fitting strategy, we choose to independently parameterise the central and secondary caustics, and make the choice that a complete given caustic is fully drawn for $0 \leq s<2$.

The abscissa $s$ is obtained by first computing the caustics with a uniform sampling in $\phi \in[0,2 \pi]$, and then computing numerically $s(\phi)$ using Eq. (8). The corresponding functions are plotted in the rightmost column of Fig. 3 for each of the three topologies. As stated before, the cusps singularities can be seen as the inflexion points of $s(\phi)$, because $(\mathrm{d} \zeta / \mathrm{d} s)_{\text {cusp }}=0$. In practice:

- in the intermediate binary case, since two branches are needed to build the upper part of the caustic, we choose $0 \leq \phi<4 \pi$, assuming that $0 \leq \phi<2 \pi$ builds the branch $\mathrm{A} \rightarrow \mathrm{C}$ and $2 \pi \leq \phi<4 \pi$ the branch $\mathrm{C} \rightarrow \mathrm{B}$. Since we 

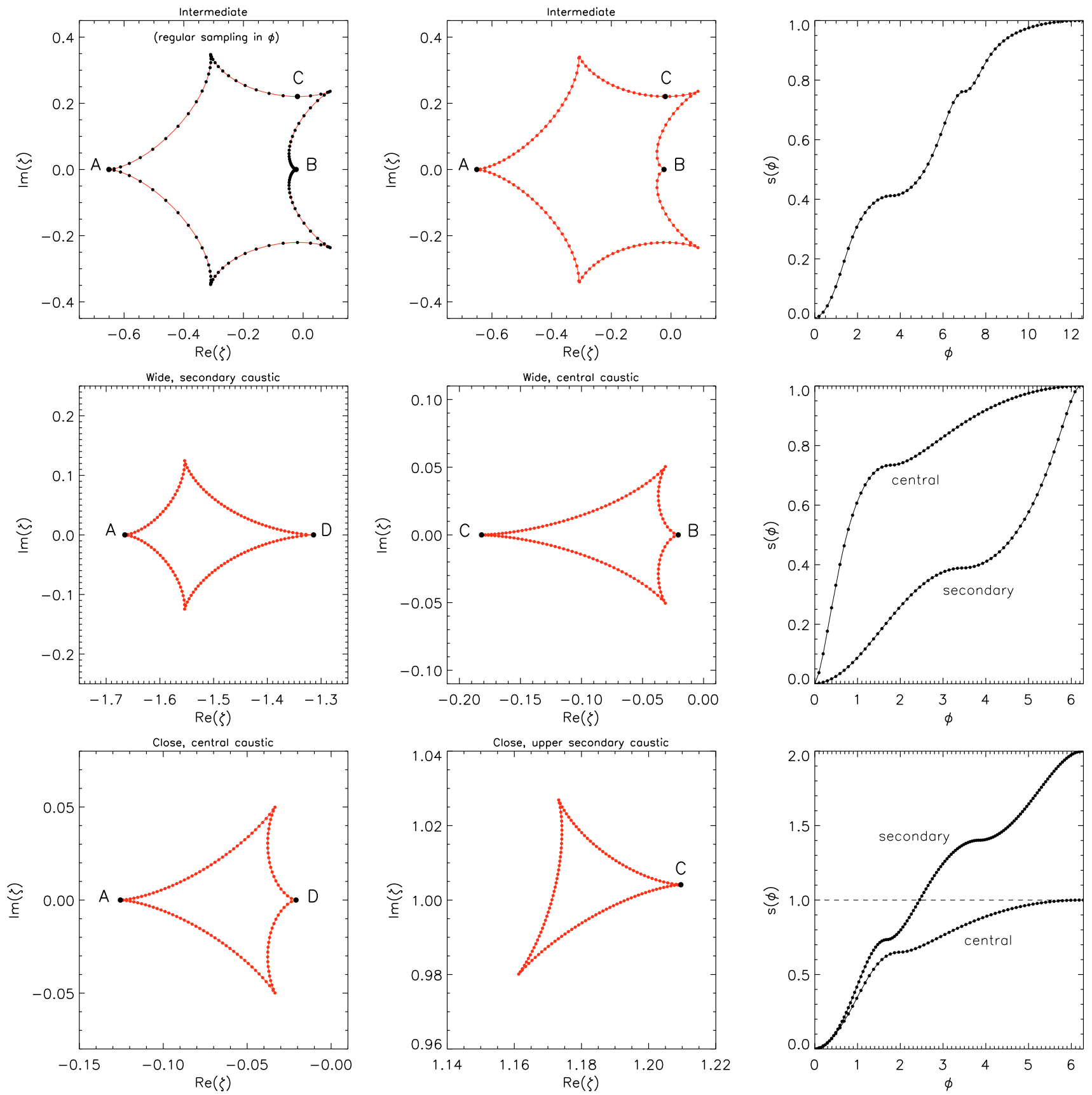

Fig. 3. The two left columns show the caustic patterns in the three possible topologies: intermediate (upper panels, $d=1.1$ and $q=0.1$ ), wide (middle panels, central and secondary caustics, $d=2$ and $q=0.1$ ) and close (central and upper secondary caustic, lower panels, $d=0.5$ and $q=0.1)$. The primary lens is at the origin of the coordinate system, with the less massive body on the left. In the upper panel on the left, the sampled points on the caustic are computed assuming a regular spacing for $\phi$ in Eq. (5), for a total of 130 points (in practice this number is larger and is adjusted to match the desired precision in the sampling). For comparison and using the same number of points, the next panel on the right shows the sampling of the caustics using a regular spacing for the curvilinear abscissa $s$ described in the paper, which results in a regular sampling of the caustics. The other plots display the wide and close caustics, also using a regular sampling for $s$. The three plots in the right most column show the function $s(\phi)$, which converts $\phi$ to $s$ according to Eq. (8) and the choices made in Sect. 3 .

have $0 \leq s<1$ for half a caustic, we set $s(\phi=0)=0$ and $s(\phi=4 \pi)=1$ in Eq. (8). The lower part of the caustic $1 \leq s<2$ is built by a horizontal reflection;

- in the wide binary case, we separately treat the central and the secondary caustic. Since in both cases a single branch is needed to build the upper part of the caustic corresponding to $0 \leq s<1$, we choose $s(\phi=0)=0$ and $s(\phi=2 \pi)=1$.
The lower part of the caustic $1 \leq s<2$ is once again built by an horizontal reflection;

- in the close binary case, we also separately parameterise the central and secondary caustics. The central caustic parameterisation is obtained in the same way as for the wide binary central caustic. On the other hand, the upper secondary caustic is fully drawn with the single branch $\mathrm{C} \rightarrow \mathrm{C}$, so that we 
have $s(\phi=0)=0$ and $s(\phi=2 \pi)=2$. The lower secondary caustic is just its horizontal reflection.

Computing and parameterising the caustics is the most time consuming part of the method. Hence an efficient way to use this parameterisation is to perform the fitting procedure on a predefined $(d, q)$ grid. This strategy is also strongly indicated when searching for all possible local minima within a reasonable $\Delta \chi^{2}$ from the global minimum, which can possibly lead to competitive solutions. For example, Kubas et al. (2005) found two local minima of almost the same goodness of fit for OGLE 2002BLG-069L, one associated with a close binary and a central caustic crossing, and the other involving a wide binary and a secondary caustic crossing.

In practice, for a given $(d, q)$ grid point we determine to which topology it corresponds (cf. Eq. (3)) and choose between the central and secondary caustics. A regular sampling of the caustics is then performed, using a regular spacing for $s$. The parameters $t_{\text {entry }}$ and $t_{\text {exit }}$ are then chosen so that during the fitting process they stay close (cf. below) to the values guessed from the light curve inspection.

\section{Fitting strategy}

\subsection{Extension to elaborated binary lens models}

A first important fact about the presented parameterisation is that it is purely geometrical, and so does not affect the way the magnification is computed. The magnification can be calculated via classical methods, such as magnification maps (Rattenbury et al. 2002; Wambsganss 1997), contouring methods (Dominik 2007; Gould \& Gaucherel 1997), improved ray shooting techniques (Dong et al. 2006) or special approximations of the magnification function. Thus, finite source effects, including limbdarkening, can be used within this framework.

Secondly, since only the positions on the caustic and their respective dates are fitted, one can consider a source trajectory affected by effects like parallax (see in particular Gould 2004) or xallarap (motion of the source due to a massive but usually unseen companion, e.g. Jiang et al. 2004; Ghosh et al. 2004). One can also consider binary lens rotation (e.g. Dong et al. 2008), by computing and parameterising the $(d, q)$ configurations at the entry and the exit dates, performing the appropriate coordinate rotation, and fitting e.g. for $\left(d_{\text {entry }}, d_{\text {exit }}, t_{\text {entry }}, t_{\text {exit }}, s_{\text {entry }}, s_{\text {exit }}\right)$.

Last, one can efficiently use the parameterisation to model events with poorly-sampled caustic-crossings (see e.g. Kains et al. 2008): it prevents the code from converging towards a minimum that does not exhibit the caustic-crossing features at the right place, which is likely to happen with the classical parameterisation. Moreover it allows us to inspect different scenarios with caustic-crossings occurring at different possible estimated dates, such as for MOA 2007-BLG-197 (Cassan et al. 2008) where the caustic entry date cannot be directly inferred from the collected data.

\subsection{Fitting using standards minimising algorithms}

When the parameterisation is used with minimising algorithms like the downhill simplex or conjugate gradient methods, which are well-suited to explore a small region around a local minimum, we choose the starting points for $t_{\text {entry }}$ and $t_{\text {exit }}$ as estimated from the light curve inspection. The starting values for $s_{\text {entry }}$ and $s_{\text {exit }}$ (both evolving in the interval $s \in[0,2]$ ), can be randomly chosen, or be set to values that allow us to explore trajectories of particular interest. A drawback of using these minimising algorithms is they may fail to locate all the minima, because they may converge to the nearest local minimum. This can be overcome by setting different starting points for $s_{\text {entry }}$ and $s_{\text {exit }}$.

Genetic algorithms (e.g. Charbonneau 1995) are quite wellsuited for use with the new parameterisation, because they combine the possibility of restricting the exploration of $t_{\text {entry }}$ and $t_{\text {exit }}$ to intervals compatible with the positions of the caustic crossings on the light curve, along with a wide exploration of all kind of trajectories by performing a dense sampling of $\left(s_{\text {entry }}, s_{\text {exit }}\right) \in$ $[0,2] \times[0,2]$. Because by essence a genetic algorithm does not converge to the exact minimum in the best found $\chi^{2}$ valley, it is recommended to further explore and refine the solution by using one of the method described above, or a Markov Chain Monte Carlo (MCMC, see below).

The new parameterisation appears also very convenient for MCMC algorithms. The starting values for the parameters $t_{\text {entry }}$ and $t_{\text {exit }}$ can be estimated from the light curve as already mentioned, while the one- $\sigma$ intervals of variation around them can be adjusted to their estimated error. One can then perform a wide exploration of all possible combinations of caustic crossing positions $s_{\text {entry }}$ or $s_{\text {exit }}$, or refine the parameters around some already found local minima, thus choosing a small corresponding $\sigma$. We find in particular that the new parameterisation greatly increases the final mean acceptance rate. MCMC also has the great advantage of providing the error bars on the best set of parameters without further calculations.

Finally, an interesting property of the parameterisation is that the event time scale, $t_{\mathrm{E}}$, is computed rather than fitted as the usual case, so that

$t_{\mathrm{E}}=\frac{t_{\text {exit }}-t_{\text {entry }}}{\left|\zeta_{\text {entry }}-\zeta_{\text {exit }}\right|}$

for a straight-line and uniform source trajectory. This formula shows that there is a strong correlation between the distance between the two caustic crossings and $t_{\mathrm{E}}$. Therefore it can happen that the minimising algorithm is not able to explore adequate values for $t_{\mathrm{E}}$, because for example the starting point was chosen too far from its final optimal value. It is then very likely to stop at a false minimum. Far from being a casual case, this is very likely to happen for binary lens caustic crossing events, for caustics show some similarity in their shape and magnification pattern over a wide range of binary separations and mass ratios (see e.g. figures in Han 2006; Chung et al. 2005; Dominik 1999b), which may draw a comparable magnification light curves, but for a range of values for $t_{\mathrm{E}}$. Using the new parameterisation, there is no such problem because $t_{\mathrm{E}}$ is not constrained, which is one of its advantages. Finally, since the best models should be associated with acceptable physical parameters, the values of $t_{\mathrm{E}}$ found for the best model(s) can be used as a consistency check of their physical plausibility.

\section{The straight line caustic approximation}

\subsection{Assumptions and application}

The magnification in the vicinity of fold caustics has been studied in detail by a number of authors (see in particular Schneider \& Weiss 1986; Gaudi \& Petters 2002; Dominik 2004), and one of the most important results in practice is that far enough from cusp singularities, the point-source magnification follows a generic behaviour. In particular, when a point-source crosses a caustic, a pair of critical images are created or merge at the critical line in the lens plane, depending on the source direction. 
At first order, their magnification is the same, and is solely dependant on the point-source distance $\zeta_{\perp}$ from the caustic, with $A \propto K / \sqrt{\zeta_{\perp}}$, where the coefficient $K$ is related to the local properties of the lens equation.

A first condition for a caustic to be treated as a straight line is that the local curvature radius of the caustic ${ }^{3}$ should be much greater than the source radius. Moreover, the gradient of the magnification along the caustic should also be negligible, which is achieved when the source crosses the caustic far enough from a cusp. Within these assumptions, semi-analytical formulae can be derived which allow a very efficient computation of the timedependant magnification during the caustic crossing, instead of the very demanding full finite-source integration.

There are many possible applications of this approximation. In order to study microlensing effects in quasar lensing, for example, Yonehara (2001) makes use of a straight line fold caustic approximation to model a suspected caustic crossing affecting image "C" of quasar Q2237+0305. In the context of stellar microlensing, Albrow et al. (1999) have worked out an improved parameterisation for binary lenses involving one well-covered caustic crossing. In this framework, the new set of parameters is directly linked to the properties of the observed caustic crossing, such as the source radius caustic-crossing time, or the date at which it happens. This method was successfully applied to a binary lens event in the Small Magellanic Cloud microlensing, MACHO 98-SMC-1 (Afonso et al. 2000). Caustic crossings are also well-suited to study source stars' brightness profiles, because different regions across their disks are differentially magnified: for example Cassan et al. (2004) used the straight line caustic approximation to study the source limb-darkening for OGLE 2002-BLG-069 without requiring any knowledge of the full binary lens model. This is of great interest when the full light curve is not well covered, or when many different binary lens models fit the light curve. If the lens is a strong binary, with typical mass ratio $q \sim 0.1-1$, the caustics have a large spatial extension, which gives the method a high potential in practice.

As mentioned in Sect. 2.2, we now examine in detail how the straight line caustic approximation can be introduced in our framework, hence providing an extension of the work initiated by Albrow et al. (1999). In the following, we consider a given and fixed binary lens configuration $(d, q)$. While outside of a caustic region a point-source is mapped onto three "non-critical" images, it is well known that a further pair of "critical" point-like images appear when the source crosses the caustic (Bourassa \& Kantowski 1975; Schneider \& Weiss 1986). The magnification for an individual image $z$ issued from a point-source located at $\zeta$ is given by

$A^{\mathrm{PS}}(z)=\left|\frac{1}{\operatorname{det} \boldsymbol{I}}\right|$,

where $\boldsymbol{I}=\partial(\xi, \eta) / \partial(x, y)$ is the Jacobian matrix associated with the lens Eq. (2), with $z=x+\mathrm{i} y$ and $\zeta=\xi+\mathrm{i} \eta$.

Under the reasonable assumption that for many cases the source size is small with respect to the caustics, the three noncritical images are weakly affected by finite source effects during a caustic crossing. On the other hand, the magnification of the two critical images is strongly dependant on the finite extension of the source. We thus divide the total magnification into two contributions,

$A\left(\zeta, \rho_{*}\right)=A_{\mathrm{NC}}\left(\zeta, \rho_{*}\right)+A_{\mathrm{C}}\left(\zeta, \rho_{*}\right)$,

3 The local curvature of the caustic can be worked out analytically using in particular Eq. (9). where $A_{\mathrm{NC}}$ stands for the three non-critical images total magnification and $A_{\mathrm{C}}$ for the two critical images total magnification, with $\rho_{*}$ the source radius in units of $\theta_{\mathrm{E}}$. We detail in the following sub-sections how we can treat $A_{\mathrm{NC}}$ and $A_{\mathrm{C}}$ in practice.

\subsection{The non-critical images magnification}

In previous studies making use of the straight line caustic approximation, the total magnification of the non-critical images is approximated at first order by the Taylor expansion $A_{\mathrm{NC}} \simeq$ $a+\omega\left(t-t_{\mathrm{c}}\right)$, where $t_{\mathrm{c}}$ is the time of the caustic crossing (in the following, we use the subscript " $c$ " for parameters computed on the caustic or critical lines, corresponding to the "entry" or "exit" used throughout the paper). Within this scheme, $a$ and $\omega$ are fitted parameters. This choice is justified in alternative parameterisations such as in Albrow et al. (1999), where only one caustic crossing is studied, or in Cassan et al. (2004) where the caustic crossing is modelled alone, assuming no knowledge of the full binary lens model. In the parameterisation presented here, it would imply adding two more parameters per caustic crossing, for a total of four more parameters in addition to $t_{\text {entry }}, t_{\text {exit }}$, $s_{\text {entry }}, s_{\text {exit }}$, while the classical full binary lens parameterisation involves only one more parameter to treat finite source effects, $\rho_{*}$ (assuming a uniformly bright source). Moreover, it can happen that for a caustic crossing located at the top of a microlensing light curve, the linear approximation of $A_{\mathrm{NC}}$ is not precise enough because of a somehow stronger local curvature in the time-dependant magnification.

Here we proceed in a different way: we choose to directly identify and track the three non-critical images during the whole caustic crossing, in order to compute their individual magnification. This can be done in the following way: at the position $\zeta_{\mathrm{c}}$ where the source centre crosses the caustic line, we use the lens Eq. (2) to locate the five images; the two critical images appear at the same position $z_{\mathrm{c}}$ on the critical curve in the lens plane, with $z_{\mathrm{c}}$ and $\zeta_{\mathrm{c}}$ satisfying Eq. (5) as well. This provides a very robust numerical test to locate the two critical images - and therefore the three non-critical images at the caustic crossing date. A NewtonRaphson method can then be implemented to "follow" the noncritical images and compute their point-source magnification

$A_{\mathrm{NC}}\left(\zeta, \rho_{*} \equiv 0\right) \simeq \sum_{i=1}^{3} A^{\mathrm{PS}}\left(z_{i}\right)$

at any position during the caustic crossing. Alternatively and for more precision, one may replace the point-source approximation by a Taylor expansion of the lens equation, and perform its integration over the source disk. Such an expansion of the magnification up to $O\left(\rho^{6}\right)$ has been studied in the general case by Pejcha \& Heyrovsky (2007), while a numerical implementation up to the hexadecapole for non-critical images has been presented in detail by Gould (2008). The derived formulae do not require a further numerical integration, thus allowing a very efficient computation of $A_{\mathrm{NC}}\left(\zeta, \rho_{*} \not \equiv 0\right)$, which is one of the main requirements about the method outlined in this section. In addition to this, Gould (2008) demonstrates that after few source radii from the caustic, the hexadecapole approximation is of very high precision.

\subsection{The critical images magnification}

For the critical images, the only relevant quantity in the straight line caustic crossing approximation is $\zeta_{\perp}$, the perpendicular distance from a point-source $\zeta$ to the caustic. Following our choice 
of curvilinear abscissa along the caustic (cf. Sect. 3), the inside region delimited by the caustic curves is always on the right hand side of the tangential vector, so that $\zeta_{\perp}>0$ inside. As shown in particular by Schneider \& Weiss (1986), the magnification of a point-like critical image in the vicinity of a caustic (and far enough from a cusp singularity) can be approximated to the first non-vanishing order by:

$A_{\mathrm{C}}^{\mathrm{PS}}\left(\zeta_{\perp}>0\right) \simeq \frac{1}{2}\left|\frac{\lambda}{\mu_{1} \sqrt{2(1-\lambda)}}\right|^{1 / 2} \frac{1}{\sqrt{\zeta_{\perp}}}$,

$A_{\mathrm{C}}^{\mathrm{PS}}\left(\zeta_{\perp} \leq 0\right)=0$,

where $\lambda=1-\partial \xi / \partial x$ and $\mu_{1}=\partial \lambda / \partial y$ are evaluated at $z=z_{\mathrm{c}}$. Within these assumptions, the two critical point-like images are of equal magnification; the corresponding total finite source magnification is thus obtained by integrating $2 \times A_{\mathrm{C}}^{\mathrm{PS}}$ over the source disk. Assuming a uniformly bright source disk, and following Schneider \& Weiss (1987), one obtains:

$A_{\mathrm{C}}\left(\zeta, \rho_{*}\right) \simeq\left|\frac{\lambda}{\mu_{1} \sqrt{2(1-\lambda)}}\right|^{1 / 2} \rho_{*}^{-1 / 2} J\left(\frac{\left|\zeta-\zeta_{\mathrm{c}}\right| \sin \psi_{\mathrm{c}}}{\rho_{*}}\right)$

where $J$ is the semi-analytical function defined in Eq. (B.7) of Schneider \& Weiss (1987) and $\zeta$ the centre of the source; $\psi_{\mathrm{c}}$ is the angle between the source trajectory and the tangent to the caustic at critical point $\zeta_{\mathrm{c}}$. In practice, $\psi_{\mathrm{c}}=\alpha-\gamma_{\mathrm{c}}$, where $\alpha$ is the trajectory angle of Fig. 2, and $\gamma_{\mathrm{c}}$ is the angle between the $x$-axis and the tangent to the caustic. Since the latter is collinear to $(\mathrm{d} \zeta / \mathrm{d} \phi)_{z_{\mathrm{c}}}$, it can be evaluated analytically using Eq. (9). A linear limb-darkened source model can also be implemented very efficiently by adding one more parameter per filter and computing further basis functions like $J$ (Albrow et al. 1999; Cassan 2005; Cassan et al. 2006).

In practice, the function $J$ can be easily tabulated to the required precision, for it only depends on the ratio $v=\zeta_{\perp} / \rho_{*}$. This is a key aspect of using the straight line caustic approximation, for one can replace a very demanding full finite-source computation by a straightforward calculation of Eq. (18), thus speeding up the computing time by many orders of magnitude. The tabulation of $J$ can be restricted to a small interval, $v=0$ to few units (see below).

\subsection{The non-caustic crossing magnification}

Outside of the caustic crossing, the point-source or a finitesource approximation like the hexadecapole is used, while their validity for a given accuracy has to be estimated. In the simplest case of the point-source approximation, for example, the finite-source magnification of a critical image rapidly converges toward the classical point-source magnification: the absolute difference between $A^{\mathrm{PS}}$ and $A_{\mathrm{C}}$ is less than $\sim 6 \times 10^{-3}$ when the source centre is farther than three source radii from the caustic, and $\sim 3 \times 10^{-4}$ for ten radii.

One way to ensure that the source crosses the caustic far enough from a cusp is to locate the curvilinear abscissa $s_{\mathrm{c}}$ as done in Sect. 2.3 and to prevent the code from exploring values of $s$ in the vicinity of $s_{\mathrm{c}}$. However if by chance the crossing occurs too close to a cusp, we expect a rather poor agreement of the magnification at the connection between the caustic crossing and non-caustic regions, which would naturally exclude the configuration obtained and thus minimise the effect of an inappropriate use of the approximation.

Finally, within the straight line caustic approximation for both the caustic entry and exit, only one parameter remains to be fitted to account for finite source effects: the source radius $\rho_{*}$, as in the classical parameterisation.

\section{Summary and prospects}

We have presented an alternative parameterisation of the caustic crossing binary lens problem, which has the main advantage of being directly linked to observed features in the light curve. We have explicitly discussed the underlying concepts and derived the corresponding equations, suggested a practical implementation, as well as examined its implications in terms of numerical robustness. Furthermore, we have shown that the straight fold caustic approximation is particularly easy to include in the presented framework, thus allowing a very high computation efficiency under some assumptions.

Current survey and follow-up networks of telescopes gather microlensing data at an accelerating rate, making the modelling a rather challenging task. Moreover, observations now involve new generations of automated telescopes, such as the RoboNet (Burgdorf et al. 2007) and RoboNet-II (Tsapras et al. 2008) initiatives, which will have in the near future the power to track many more microlensing events. In this respect, a more automated modelling tool would be helpful to catch up with the flow of incoming data. Indeed, such a requirement for more automated software also applies for telescopes based in isolated or remote locations, like Dome $\mathrm{C}$ in Antarctica (see for example prospects underlined in Beaulieu et al. 2005), where microlensing would benefit from a continuous monitoring of the Galactic bulge in addition to the site's climatic advantages.

The automation of algorithms dedicated to microlensing event prioritising has seen important efforts (Dominik et al. 2007; Snodgrass et al. 2008), but similar progresses in modelling tasks remain to be achieved on the mathematical and numerical sides to keep up with the increasing rate of detection of anomalous microlensing events, which is one of the motivations of this work.

Acknowledgements. I would like to thank N. Kains, D. Kubas, C. Snodgrass and Y. Tsapras for very valuable comments on the manuscript, as well as the anonymous referee whose comments and suggestions helped improve the present work. I also thank A. Yonehara and R. W. Schmidt for inspiring discussions on caustic topologies and properties. I thank K. Horne for inviting me for a visit at SUPA, University of St. Andrews, where this work was initiated. I acknowledge the ESO Director General Discretionary Found (DGDF) for financing a onemonth visitor stay at ESO Santiago in March 2008, as well as S. Brillant and D. Kubas for the invitation.

\section{References}

Afonso, C., Alard, C., Albert, J. N., et al. 2000, ApJ, 532, 340

Albrow, M. D., Beaulieu, J.-P., Caldwell, J. A. R., et al. 1999, ApJ, 522, 1022

Batista, V., Dong, S., Udalski, A., et al. 2008, in preparation

Beaulieu, J.-P., Bennett, D. P., Fouqué, P., et al. 2006, Nature, 439, 437

Beaulieu, J. P., Cassan, A., Kubas, D., et al. 2005, in EAS Publ. Ser., ed. M.

Giard, F. Casoli, \& F. Paletou, 14, 297

Bennett, D. P., \& Rhie, S. H. 2002, ApJ, 574, 985

Bennett, D. P., Bond, I. A., Udalski, A., et al. 2008, ApJ, 684, 663

Bond, I. A., Abe, F., Dodd, R. J., et al. 2001, MNRAS, 327, 868

Bond, I. A., Udalski, A., Jaroszyński, M., et al. 2004, ApJ, 606, L155

Bourassa, R. R., \& Kantowski, R. 1975, ApJ, 195, 13

Burgdorf, M. J., Bramich, D. M., Dominik, M., et al. 2007, Planet. Space Sci., 55,582

Cassan, A. 2005, Ph.D. Thesis, Institut d'Astrophysique de Paris/Université Pierre et Marie Curie, Paris VI

Cassan, A., Beaulieu, J. P., Brillant, S., et al. 2004, A\&A, 419, L1

Cassan, A., Beaulieu, J.-P., Fouqué, P., et al. 2006, A\&A, 460, 277

Cassan, A., Kubas, D., Bond, I., et al. 2008, in preparation 
Charbonneau, P. 1995, ApJS, 101, 309

Chung, S.-J., Han, C., Park, B.-G., et al. 2005, ApJ, 630, 535

Dominik, M. 1999a, A\&A, 341, 943

Dominik, M. 1999b, A\&A, 349, 108

Dominik, M. 2004, MNRAS, 353, 69

Dominik, M. 2007, MNRAS, 377, 1679

Dominik, M., Rattenbury, N. J., Allan, A., et al. 2007, MNRAS, 380, 792

Dong, S., DePoy, D. L., Gaudi, B. S., et al. 2006, ApJ, 642, 842

Dong, S., Gould, A., Udalski, A., et al. 2008, ArXiv e-prints, 804, 1354

Einstein, A. 1936, Science, 84, 506

Erdl, H., \& Schneider, P. 1993, A\&A, 268, 453

Gaudi, B. S., \& Petters, A. O. 2002, ApJ, 574, 970

Gaudi, B. S., Albrow, M. D., An, J., et al. 2002, ApJ, 566, 463

Gaudi, B. S., Bennett, D. P., Udalski, A., et al. 2008, Science, 319, 927

Ghosh, H., DePoy, D. L., Gal-Yam, A., et al. 2004, ApJ, 615, 450

Gould, A. 2004, ApJ, 606, 319

Gould, A. 2008, ApJ, 681, 1593

Gould, A., \& Gaucherel, C. 1997, ApJ, 477, 580

Gould, A., Udalski, A., An, D., et al. 2006, ApJ, 644, L37

Han, C. 2006, ApJ, 638, 1080

Jaroszynski, M., Udalski, A., Kubiak, M., et al. 2004, Acta Astron., 54, 103 Jaroszynski, M., Udalski, A., Kubiak, M., et al. 2005, Acta Astron., 55, 159 Jiang, G., DePoy, D. L., Gal-Yam, A., et al. 2004, ApJ, 617, 1307
Kains, N., Cassan, A., Horne, K., et al. 2008, submitted

Kubas, D., Cassan, A., Beaulieu, J. P., et al. 2005, A\&A, 435, 941

Kubas, D., Cassan, A., Dominik, M., et al. 2008, A\&A, 483, 317

Mao, S., \& Paczynski, B. 1991, ApJ, 374, L37

Mayor, M., \& Queloz, D. 1995, Nature, 378, 355

Paczynski, B. 1986, ApJ, 304, 1

Pejcha, O., \& Heyrovsky, D. 2007, ArXiv e-prints, 712

Rattenbury, N. J., Bond, I. A., Skuljan, J., \& Yock, P. C. M. 2002, MNRAS, 335, 159

Schneider, P., \& Weiss, A. 1986, A\&A, 164, 237

Schneider, P., \& Weiss, A. 1987, A\&A, 171, 49

Snodgrass, C., Horne, K., \& Tsapras, Y. 2004, MNRAS, 351, 967

Snodgrass, C., Tsapras, Y., Street, R., et al. 2008, in Manchester Microlensing Conference, [arXiv: 0805.2159]

Tsapras, Y., Horne, K., Kane, S., \& Carson, R. 2003, MNRAS, 343, 1131

Tsapras, Y., Street, R., Horne, K., et al. 2008, ArXiv e-prints, 808, 813, submitted Udalski, A. 2003, Acta Astron., 53, 291

Udalski, A., Szymanski, M., Mao, S., et al. 1994, ApJ, 436, L103

Udalski, A., Jaroszyński, M., Paczyński, B., et al. 2005, ApJ, 628, L109

Wambsganss, J. 1997, MNRAS, 284, 172

Witt, H. J. 1990, A\&A, 236, 311

Wolszczan, A., \& Frail, D. A. 1992, Nature, 355, 145

Yonehara, A. 2001, ApJ, 548, L127 